TITLE:

\title{
Optimization methodology of operation of orifice-shaped micromixer based on micro-jet concept
}

\section{AUTHOR(S):}

Matsuyama, Kazuo; Mine, Koji; Kubo, Hideaki; Aoki, Nobuaki; Mae, Kazuhiro

\section{CITATION:}

Matsuyama, Kazuo ... [et al]. Optimization methodology of operation of orifice-shaped micromixer based on micro-jet concept. Chemical Engineering Science 2010, 65(22): 5912-5920

ISSUE DATE:

2010-11-15

URL:

http://hdl.handle.net/2433/130707

\section{RIGHT:}

(C) 2010 Elsevier Ltd; この論文は出版社版でありません。引用の際には 出版社版をご確認ご利用ください。; This is not the published version. Please cite only the published version. 
[Full Length Paper]

Optimization methodology of operation of orifice-shaped micromixer

based on micro-jet concept

Kazuo Matsuyama ${ }^{\mathrm{a}}$, Koji Mine ${ }^{\mathrm{a}}$, Hideaki Kubo ${ }^{\mathrm{a}}$, Nobuaki Aoki ${ }^{\mathrm{b}}, \mathrm{Kazuhiro}_{\text {Mae }}{ }^{\mathrm{b} *}$

${ }^{\mathrm{a}}$ Global R\&D - Processing Development, Kao Corporation, Minato, Wakayama 640-8580,

Japan

${ }^{\mathrm{b}}$ Department of Chemical Engineering, Graduate School of Engineering, Kyoto University,

Kyoto-daigaku Katsura, Nishikyo-ku, Kyoto 615-8510, Japan

* Corresponding author. Tel.: +81 75383 2668; fax: +81 753832658.

E-mail address: kaz@cheme.kyoto-u.ac.jp (K. Mae) 


\section{Abstract}

To establish an optimization methodology for the emulsification process in microchannels, we have investigated the relationship between the channel geometry of micromixers and the size of the formed droplets. We focus the channel geometry of orifice including the sudden contraction and expansion of the flow. The experimental results indicate that the channel geometry is effective in producing fine droplets, and that the mean droplet diameter is predicted on the basis of the pressure drop due to convection and the energy dissipation rate in the mixer chamber irrespective of the orifice geometry. In the orifice-shaped micromixer, the kinetic energy given to a fluid by contraction is dissipated within the order of milliseconds by the formation of a jet flow of the order of several hundreds of micrometers; we have named this jet "micro-jet." On the basis of this mechanism, the energy dissipation rate in a micro-jet, $\varepsilon_{\text {jet }}$, is quantified. The mean droplet diameter is proportional to $\varepsilon_{\text {jet }}{ }^{-0.4}$ irrespective of the channel geometry of the mixer. Using this formulation, we have integrated the geometric parameters of the orifice and the physical properties of the fluids into the expression of $\varepsilon_{\text {jet }}$ to establish an optimization methodology of the droplet formation in the orifice-shaped micromixer.

Keywords: Micromixer; Channel geometry; Emulsion; Optimization; Turbulence; Energy 
Dissipation 


\section{Introduction}

In microchannels, the rapid mixing of two or more fluids is achieved because of the short distance between the fluids, and the rate of the mass transfer between the immiscible phases is enhanced because of the high surface-to-volume ratio of these fluids. By leveraging these advantages, researchers have developed microdevices, including channels of the order of sub-millimeters for reaction (Maruyama et al., 2003), extraction (Benz et al., 2001), and phase separation (Freitas et al., 2003). In conventional research, the focus is to propose a device itself and to optimization parameters such as the size and the geometry of channels; the operating conditions are determined intuitively. However, microdevices developed in an intuitive manner lack versatility. For the use of microdevices in industrial production, it is necessary to establish an optimization methodology based on an optimization equation and indices expressing the characteristics of the phenomena in the device.

In order to carry out mixing in a homogeneous phase, relationships between the optimization parameters of the mixer and the mixing performance have been studied, and indices for the mixer optimization have been proposed. Kockmann et al. (2006) have evaluated the convective mixing rate in micromixers with various microchannel geometries by using computational fluid dynamics (CFD) simulations and the selectivity of instantaneous parallel reactions (called Villermaux/Dushman reaction) (Guichardon and Falk, 2000; 
Guichardon et al., 2000). On the basis of these evaluations, the mixing rate has been correlated with the throughput and the pressure drop. In order to derive the relationship between the channel geometry, the operating conditions, and the mixing performance of a micromixer, Falk et al. (2010) have calculated the energy dissipation rates $(\varepsilon)$ in mixing chambers with various geometries as an index of the micromixer optimization and determined the relationship between the mixing time estimated from the result of the Villermaux/Dushman reaction and the energy dissipation rate.

In addition to homogeneous mixing, mixing devices are widely used in batch and continuous processes, including multiphase operations (e.g., emulsification and blending). In a conventional process, the turbulence in a rotor-stator mixer or a static mixer is generally applied to the emulsification process. By using the turbulence theory, Davies (1985) and Middleman (1974) formulated the energy dissipation rate in the droplet dispersion caused by convective mixing. Davies has expressed the maximum size of the droplets generated by a convective eddy, $d_{\max }$, as a function of the energy dissipation rate $\varepsilon\left[\mathrm{W} \cdot \mathrm{kg}^{-1}\right]$ as follows:

$$
d_{\max }=\text { const. } \cdot\left(\sigma / \rho_{\mathrm{c}}\right)^{0.6} \varepsilon^{-0.4}
$$

where $\sigma$ is the interface tension $\left[\mathrm{N} \cdot \mathrm{m}^{-1}\right]$ and $\rho_{\mathrm{c}}$ is the density of the continuous phase $\left[\mathrm{kg} \cdot \mathrm{m}^{-3}\right]$. As shown in Eq. 1, the droplet diameter is generally proportional to $\varepsilon^{-0.4}$, and this is the case in various formulations (Paul et al., 2004). Middleman has macroscopically defined $\varepsilon$ as

$$
\varepsilon=\frac{Q \cdot \Delta P}{\rho \cdot V}
$$


where $Q$ is the volume flow rate $\left[\mathrm{m}^{3} \cdot \mathrm{s}^{-1}\right], \Delta P$ is the pressure drop in the focused space $[\mathrm{Pa}], \rho$ is the density of the fluid $\left[\mathrm{kg} \cdot \mathrm{m}^{-3}\right]$, and $V$ is the volume of the focused space $\left[\mathrm{m}^{3}\right]$. Eq. (2) is also expressed using the space time $\tau$ [s] as follows:

$$
\varepsilon=\frac{\Delta P}{\rho \cdot \tau}
$$

Eq. 3 implies that the rate of change in the pressure drop dominates the value of $\varepsilon$ for a fixed operating fluid. With a relatively low value of $\Delta P$, a high energy dissipation rate can be obtained in the microchannels. $\tau$ in microchannels is considerably small as discussed in the following section.

Microdevices have a potential to change the policy for process development. The advantages of microdevices include the relatively short mixing time of the order of milliseconds as compared to that (of the order of seconds) in a beaker. Such a short-time operation provides a possibility to produce novel products since the mixing time, for example, can be of the same timescale as a phase transition such as nucleation. Therefore, microdevices determine the quality of products. In addition, microdevices offer opportunities for equipment miniaturization, energy saving, and reduction of wastes (Charpentier, 2005).

Investigations on the emulsification process carried out using micromixers have been extensively conducted. Soap-free emulsification was carried out using a micromixer on the basis of the split and the recombination of fluid streams (Mae et al., 2004). In this mixer, the oil phase (dispersed phase) interacted with the channel wall, and this interaction enabled 
soap-free emulsification. For a micromixer that is based on a split fluid having a lamellar structure and on the fluid contraction, the energy consumption in the mixer has been compared with that of a batch stirrer (Bayer et al., 2004), and the relationship between the operating conditions and the mixing performance has also been studied (Löb et al., 2006). However, in contrast with the reaction and the extraction operations, a few papers (e.g., production of monodispersed emulsification (Kawakatsu et al., 1997) and microcapsule (Utada et al., 2005)) have explicitly declared the advantages of a micromixer with respect to the emulsification process; further, the range of the applied operating conditions is limited to low Reynolds numbers.

In our previous work, the effect of the channel geometry of conventional micromixers on the emulsification performance (e.g., mean droplet diameter and droplet size distribution) was evaluated (Mae et al., 2004). The results suggested that the velocity in the outlet channel as well as the number of stages was a crucial factor to obtain a uniform emulsion. Löb et al. (2006) have investigated liquid/liquid-dispersion in interdigital micromixers with a slit-shaped microchannel. The results suggested that the growing hydrodynamic focusing at the slit-shaped microchannel reduced the droplet size. By integrating these results in the development of micromixer, a highly effective mixing devise for emulsification process can be devised.

In this context, we propose a novel orifice-shaped micromixer including the sudden 
contraction and expansion of the flow for carrying out the emulsification process. The detailed effects of the physical properties of the fluid and the optimization parameters of the mixer, such as channel size, flow rate, channel geometry, and droplet diameter, are evaluated using the indices that represent the characteristics of the flow inside of the micromixers. On the basis of this evaluation, an optimization methodology is established for expressing the relationship between the droplet size and the optimization parameters. 


\section{Experimental}

\subsection{Droplet production using orifice-shaped micromixers with various geometric parameters}

Fig. 1 illustrates the geometry and the geometric parameters of an orifice-shaped micromixer made of stainless steel (SUS316). The geometry consists of a confluence of a coaxial double-pipe and a pinhole-like channel. The channel length between the sudden contraction and the expansion is very short. The orifice is used as a short, narrow channel, which connects to the wide channel, and the pinhole channel can be regarded as the orifice. Since geometry is a distinctive feature of this micromixer, the mixer was named “orifice-shaped micromixer."

Table 1 summarizes the geometric parameters and operating conditions of the orifice-shaped micromixers used in this study. The angle of contraction, $\theta$, the orifice hole diameter and length, $d$ and $L$, and the channel diameter after sudden expansion, $D_{\text {exit }}$, are the focused geometric parameters. The emulsification performance (e.g., mean droplet diameter and droplet size distribution) of these mixers was evaluated. Six orifice-shaped mixers with different geometric parameters were fabricated, and the ratio of the orifice length $L$ to the orifice hole diameter $d$ was approximately 3 for all the six mixers. The variation in each geometric parameter was not constant because ready-made fittings were used for building the 
orifices of some mixers (No.1, 2, 4). The flow rate ranged from 3 to $45 \mathrm{~L} \cdot \mathrm{h}^{-1}$, which is a relatively large value for single-channel processes carried out using micromixers. This range was determined so that the experimental setups installing the micromixers have a common operating range of the total pressure drop in the entire micromixer channel $\Delta P$ and the Reynolds number calculated from the orifice hole diameter, the average velocity in the orifice hole, the density of the mixture fluid, and the viscosity of the continuous phase.

Table 2 lists the compositions of the solutions used in the emulsification process. A cyclic silicone (cyclopentasiloxane, Shin-Etsu Chemical) was used as the oil phase (dispersed phase), resulting in the $\mathrm{O} / \mathrm{W}$-type emulsion. In general, this type of emulsion is versatile and has low viscosity. For these reasons, this type of emulsion was selected as a model case of emulsification. Cyclic silicone was chosen because it is widely used in cosmetics and has almost no polarity and a considerably lower solubility in water than that in the other oil solutions. Therefore, the effects of the mutual solubility of the oil and the water phases are negligible, and the low solubility facilitates the identification of the effects of the channel geometry on the droplet formation. As the water phase (continuous phase), a polymer dispersant, polyvinyl alcohol (Nikko Chemicals), was used for preventing the coalescence of the produced droplets and to enhance the accuracy in the measurement of the droplet diameter. It was also confirmed that the droplet diameter remained unchanged between the emulsification experiments and the measurement. In the experiments, two aqueous solutions 
C-1 and C-2 with different viscosities were used as the water phase. The viscosity of the water phase was increased by adding a polysaccharide emulsifier stabilizer (Sodium stearoxy polysaccharide sulfonate, Kao Corporation). When C-1 was used as the water phase, the mass fraction of the dispersed oil phase was 30\%; and when C-2 was used, the fraction was $20 \%$.

Fig. 2 shows a schematic representation of the experimental setup. The two solutions of the water and oil phases were fed by two diaphragm pumps with low pulsation (TPL, Tacmina Corporation). These pumps enable constant rate feeds even for low-viscosity fluids and a maximum high pumping pressure of $3 \mathrm{MPa}$. The pressure drop between each inlet of the two phases and the channel outlet was measured using two pressure transducers (PMP41, Endress+Hauser) installed into the inlet channels (the transducers are denoted by PI as shown in Fig .2). The flow-rate-weighted average of measured pressure drops of the two phases was used as the total pressure drop in the entire micromixer channel. The emulsified solution was sampled at the exit of the outlet channel connected to the micromixers. The diameter of the emulsified droplets was measured using a particle size distribution analyzer based on laser diffraction and scattering (LA-910, Horiba). In the measurements, the relative refractive index was set to 1.06 .

\subsection{Precipitation of fine particles using micromixers}


An instantaneous precipitation of fine particles was carried out by mixing a solution and a poor solvent in order to verify the effectiveness of the key index in the optimization of the micromixer for the emulsification process. The mixing rate and the uniformity in the mixing chamber were evaluated on the basis of the particle size distribution (Nagasawa et al., 2005). In the experiment, $5 \mathrm{wt} \%$ 1-octadecanol (Kao Corporation) solution in ethanol was adopted as a model solution. As a poor solvent, $1 \mathrm{wt} \%$ surfactant water solution (TWEEN80, Kao Corporation) was used. The particles of 1-octadecanol formed easily because of the low solubility in water $\left(1 \mathrm{mg} \cdot \mathrm{m}^{-3}\right)$ and the rapid nucleation of 1-octadecanol. In cosmetics, 1-octadecanol is widely employed as a raw material and used as a lipid crystal. The solution of 1-octadecanol and the poor solvent were fed into the micromixers, and the fine particles of 1-octadecanol were sampled. The ratio of the flow rate of the poor solvent to that of the solution of 1-octadecanol was fixed at 3 . The experimental setup and the particle size distribution analyzer were the same as those described in the previous section. In the measurements, the relative refractive index was set to 1.2.

For verifying the usefulness of the orifice-shaped micromixer, the following micromixers were used: the tee-shaped micromixer $\left(\right.$ Swagelok $\left.^{\circledR}\right)$ whose outlet-channel diameter $\times$ length was $0.3 \mathrm{~mm} \times 0.9 \mathrm{~mm}$, the tube-shaped micromixer whose straight tube channel diameter $\times$ length was $0.5 \mathrm{~mm} \times 24 \mathrm{~mm}$, the orifice-shaped micromixer (No. 2, see Table 1), and the bend-shaped micromixer whose outlet-channel diameter $\times$ length was 0.4 
$\mathrm{mm} \times 3 \mathrm{~mm}$. The precise channel geometries and the schematic representation of conventional mixers except the tee-shaped micromixer are given in Figs. 1 and 3 and in Matsuyama et al. (2008). The flow rate ranged from 3.6 to $7.2 \mathrm{~L} \cdot \mathrm{h}^{-1}$ for the tee-shaped micromixer, 6.3 to $18.3 \mathrm{~L} \cdot \mathrm{h}^{-1}$ for the tube-shaped micromixer, 6.3 to $8.8 \mathrm{~L} \cdot \mathrm{h}^{-1}$ for the orifice-shaped micromixer, and 4.5 to $8.2 \mathrm{~L} \cdot \mathrm{h}^{-1}$ for the bend-shaped micromixer.

\section{Results and discussion}

In order to develop microdevices appropriate for carrying out the emulsification process in actual production, an optimization method for micromixers capable of producing the desired droplet size is required. For this purpose, the effects of the operating conditions and the optimization parameters of orifice-shaped micromixers with the identified geometry on the emulsification performance are discussed, and the key index in the optimization of micromixers for carrying out the emulsification process, the energy dissipation rate, is derived. Then, the effectiveness of the energy dissipation rate in the micromixer optimization is verified through experiments related to the precipitation of fine particles. 


\subsection{Effects of geometric parameters of orifice-shaped micromixers}

\subsubsection{Effect of ratio of channel size expansion}

From the comparison of the orifice-shaped micromixers listed in Table 1, the droplet size and the droplet size distribution of mixer No. 1 was the largest and the broadest, respectively, as shown in Figs. 4(a) and 4(b). This result suggests that a large expansion ratio of the channel width at the exit of the orifice geometry is required for the formation of fine droplets with a narrow particle size distribution, which is a required quality of commercial products. Hereafter, we proceed with the study of the effects of channel geometries of the orifice-shaped micromixers No. 2-6 on the emulsification process.

\subsubsection{Evaluation of emulsification performance of orifice-shaped micromixers on the basis of pressure drop}

For establishing an optimization methodology of orifice-shaped micromixers for the emulsification process, the pressure drop effect of the channel contraction and expansion on the emulsification process is considered. In the region of the flow rate considered in this study, the shear force dominated the flow as compared to the interfacial tension, and the convective eddy controlled the droplet formation in the microscale space in the same manner as that in a macroscale space. Following this discussion, the pressure drop due to convection can be 
considered to be a candidate for the index expressing the dissipation of energy required for generating a convective eddy. The mean droplet diameter obtained by using the orifice-shaped micromixers No. 2-6 with various total flow rates and the two solutions is plotted as a function of the total pressure drop in the entire micromixer $\Delta P$, as shown in Fig. 5. Note that $\Delta P$ corresponded approximately to the pressure drop caused by convection in the orifice-shaped micromixers since the pressure drop in the orifice part dominated the overall pressure drop because of the channel width of this part was considerably smaller than that of the other parts. As the mean droplet diameter, the Sauter diameter, $D_{32}=\Sigma D_{i}^{3} / \Sigma D_{i}^{2}\left(D_{i}\right.$ : diameter of droplet $i$ ), was calculated on the basis of the surface area of the droplets. The Sauter diameter is often adopted in multiphase mixing and atomization. Hereafter, we use the Sauter diameter as the measure of the emulsification performance of the micromixers. At fixed compositions of the solutions, the result shown in Fig. 5 indicated that $\Delta P$ determined the droplet diameter irrespective of the orifice geometry. Therefore, it was confirmed that the pressure drop caused by the sudden flow contraction and expansion is effective as an index for predicting the droplet diameter.

\subsubsection{Effectiveness of orifice-shaped micromixers for emulsification in actual production}

As shown in the previous section, the droplet diameter was predicted from the pressure drop through the sudden flow contraction and expansion. In other words, the 
pressure drop, the dissipation of the kinetic energy of the flow, was converted into a convective eddy, and the formed eddy dominated the droplet generation. Therefore, the flow phenomena for the emulsification in the micromixers were the same as those in macroscale channels. However, the orifice-shaped micromixer has an advantage that the measured pressure drop determines the phenomena and the produced droplet diameter irrespective of the channel geometries. Because the real-time pressure in actual processes is usually measured, the droplet diameter can be monitored online, as one of indices of product quality as well as the state of the operation in the process. From the change in the pressure value over time caused by the ablation brought about by long-term use, the time for replacing the units of the mixer can also be determined. The mixer performance is maintained by adjusting the pressure value by changing the operating conditions such as the flow rate. Moreover, the axisymmetric geometry of the orifice-shaped mixer contributes to the ease of fabrication and maintenance of mixers. Thus, in addition to fine emulsification, the orifice-shaped micromixer is useful in industrial production from the viewpoints of fabrication, maintenance, and process monitoring.

\subsection{Optimization methodology of micromixers based on the mechanism of droplet formation in} microspace 


\subsubsection{Micro-jet at the sudden expansion in orifice-shaped micromixers}

Through the discussion in Section 3.1, we have correlated the droplet diameter and the pressure drop in micromixers. The pressure drop determined the overall phenomenon of droplet formation. In order to derive an optimization methodology that determines the optimization parameters of micromixers for emulsification, such as channel sizes and operating conditions, an index for precisely describing the phenomenon of droplet formation is necessary.

For this purpose, first, the mechanism of the energy dissipation caused by the flow contraction and its sudden expansion into a convective eddy formation is discussed. In an orifice, the flow accelerates and gains kinetic energy because of a channel contraction, and energy is consumed by a sudden expansion of the flow brought about by the decrease in the flow velocity and the formation of the convective eddy. This flow phenomenon is called a jet flow and is shown in Fig. 6. A jet injected from the orifice is subjected to a shear force from the surrounding fluid, resulting in the generation of a local convective eddy inside of the jet. The jet flow was investigated a long time ago, and Rajaratnam (1976) established the basic principles of jet flow. The jet is divided into the following two regions on the basis of the distance from the exit of the orifice (orifice hole): the near-field where the shear is enhanced suddenly and the developed-field where a turbulent flow develops. Inside of the near-field, a region called a potential core exists from the exit of the orifice to a point whose distance from 
the exit is approximately six times the orifice diameter (denoted as $6 d$ ). In the potential core, the flow is not subjected to shear. The mixing and the dispersion in a jet flow have also been widely investigated. Baldyga et al. (1994) have evaluated the mixing performance inside of a jet by using rapid parallel reactions. They have formulated the distribution of the energy dissipation rate inside of a jet, $\varepsilon\left[\mathrm{W} \cdot \mathrm{kg}^{-1}\right]$. In the formulation, $\varepsilon$ maximizes at the point $6 d-7 d$ away from the nozzle outlet ( $d$ is the nozzle hole diameter) and then declines sharply. Zumaeta et al. (2008) have applied the jet flow to the breakage of protein precipitates and investigated the relation between $\varepsilon$ and the operating conditions obtained from the CFD simulations and experiments. Macloughlin et al. (1998) have expressed the rate of cell death as a function of the mean value of $\varepsilon$. This mean value is calculated by integrating $\varepsilon$ in the region $6 d$ to $16 d$ away from the exit of the hole, where strong convection occurs. At a point $16 d$ away from the hole, the value of $\varepsilon$ is approximately one-fiftieth of its maximum.

On the basis of the above findings, the mechanism of droplet formation in the orifice-shaped micromixer is then identified. The theory of jet flow is valid irrespective of the channel size. Therefore, the theory can be applied to the discussion of a sub-millimeter-sized jet. On the basis of this theory, the velocity distribution inside of a jet is fixed at a constant initial velocity, $u_{0}\left[\mathrm{~m} \cdot \mathrm{s}^{-1}\right]$, irrespective of the diameter of the orifice hole. A parameter that changes in accordance with the size ought to be focused on. For this purpose, the space time, $\tau_{\text {jet }}[\mathrm{s}]$, defined by the following equation is suitable: 


$$
\tau_{\text {jet }}=V_{\text {jet }} / Q
$$

where $V_{\text {jet }}$ is the volume of the space in which the shear is applied $\left[\mathrm{m}^{3}\right]$ and $Q$ is the volume flow rate $\left[\mathrm{m}^{3} \cdot \mathrm{s}^{-1}\right]$. In the case of a fixed $u_{0}, V_{\text {jet }}$ is proportional to the cube of the diameter of the orifice hole and $Q$ is proportional to the square of the diameter. In other words, $\tau_{\text {jet }}$ is directly proportional to the hole diameter and is one to three orders of magnitude smaller than that in the case of the macroscale channels. In order to quantify the value of $\tau_{\text {jet }}$, the value of $V_{\text {jet }}$ is defined as shown in Fig. 7. The length and the width of the jet are defined on the basis of the research results and the existing theory of jet flow. In this definition, the volumes of the orifice hole and the potential core are included in $V_{\text {jet }}$. For example, under the condition that the hole diameter is $500 \mu \mathrm{m}$ and the volume flow rate is $20 \mathrm{~L} \cdot \mathrm{h}^{-1}, V_{\text {jet }}$ is $8 \mathrm{~mm}^{3}$ and $\tau_{\text {jet }}$ is 1.4 ms. This value of $\tau_{\text {jet }}$ implies that the time for applying shear to the fluid is in the order of milliseconds because of the miniaturization of the space for the jet to the order of micrometers. This value also implies that the convective mixing is completed within a few milliseconds. Therefore, the mechanism of droplet formation in the orifice-shaped micromixer is as follows: the kinetic energy of the fluid upon contraction is dissipated within a period of milliseconds because of the jet flow that is in the order of micrometers, that is, micro-jet. In other words, the emulsification is dominated by the energy dissipation rate. The operation resulting in a high rate of energy dissipation enables the emulsification with a single pass and a single mixer. As discussed here, a high energy dissipation rate is achieved in the micrometer-sized orifice. 
This is an advantage of using micromixers for the emulsification process.

\subsubsection{Definition of energy dissipation rate in micro-jet}

The value of $\varepsilon$ calculated from Eq. (3) is an average for the considered space and is dependent on the focused space. Here, the part of the microchannel that contributes to the emulsification in the orifice-shaped micromixer is the focused space. The volume of this space is considered to be equal to $V_{\text {jet }}$ defined in Fig. 7. In this subsection, the representative value of $\varepsilon$ in the orifice-shaped micromixer, $\varepsilon_{\text {jet }}$, is obtained on the basis of the pressure drop in the volume. Then, an optimization methodology is derived by expressing the droplet diameter as a function of $\varepsilon_{\text {jet }}$ in the next subsection.

The pressure drop for deriving an expression of $\varepsilon_{\text {jet }}$ is then quantified. The general expression of $\Delta P$ related to the orifice geometry is given as

$$
\Delta P=\zeta \frac{1}{2} \rho u_{0}^{2}
$$

where $\rho$ is the density $\left[\mathrm{kg} \cdot \mathrm{m}^{-3}\right] \cdot u_{0}$ is the average velocity in the orifice $\left[\mathrm{m} \cdot \mathrm{s}^{-1}\right]$ and is the same as the initial velocity of the jet. The coefficient $\zeta$ depends on the geometry of the orifice, and its value is usually between 1.2 and 2. From the result shown in Fig. 5, the values of $\zeta$ in the case of the orifice-shaped micromixers No. 2-6 were determined as shown in Fig. 8. Fig. 8 also indicates that this parameter depended only on the orifice geometry and was independent of the viscosity of the solution. This independence also implies that the contribution of the pressure drop due to the friction between the fluid and the wall could be 
ignored. In the case of mixer No. $2, \zeta$ was less than 1 and was considerably smaller than that of the other mixers. This is because the ratio of the channel expansion was small and the kinetic energy gained by the contraction was not dissipated efficiently in the micro-jet. The results indicate that a large expansion ratio is required for achieving the pressure loss coefficient $\zeta \geq 1$, which implies that the kinetic energy of the flow in the orifice is effectively used in the emulsification process.

Finally, from the obtained expressions of $V_{\text {jet }}$, the parameter related to the orifice geometry $\zeta$, and Eq. (2), $\varepsilon_{\text {jet }}$ was derived as follows:

$$
\varepsilon_{\text {jet }}=\frac{\pi d^{2} / 4 \cdot u_{0} \cdot \zeta \frac{1}{2} \rho u_{0}^{2}}{\rho \cdot 81.6 d \cdot \pi d^{2} / 4}=0.0061 \zeta \frac{u_{0}^{3}}{d} .
$$

\subsubsection{Optimization methodology of orifice-shaped micromixers}

Fig. 9 shows the mean droplet diameter from Fig. 5 as a function of the energy dissipation rate defined by Eq. (6) in a double-logarithmic plot. For the four geometries of orifice-shaped micromixers No. 3-6, the droplet diameter was expressed only by $\varepsilon_{\text {jet. }}$. Therefore, the channel size and the operating conditions of the micromixers were optimized for emulsification using $\varepsilon_{\mathrm{jet}}$. The droplet diameter was proportional to $\varepsilon_{\mathrm{jet}}{ }^{-0.4}$, and this relation indicated that the theory of turbulent flow was applicable to the discussion of the mechanism of droplet formation in a micro-jet.

Next, the effect of the composition of solutions was examined. In the experiments, 
the orifice-shaped micromixers No. 3-6 that had sufficiently large ratios of channel expansion were employed. The compositions of the solutions were those of C-1 and C-2 shown in Table 2. Fig. 10 shows the relationship between the mean droplet diameter and the energy dissipation rate of the two solutions. Irrespective of the composition of the solutions and the geometric parameters, the mean diameter was proportional to $\varepsilon_{\text {jet }}^{-0.4}$. The physical properties of the dispersed oil phase directly affected the formation of the droplet and the droplet size (e.g., interface tension and density) and influenced the coalescence of the formed droplets (e.g., interface tension and viscosity). The volume fraction of the oil phase also affected the coalescence. Moreover, under the condition that the energy dissipation rate was sufficiently large, the physical properties of the continuous phase affected the droplet diameter since the droplet diameter reached the smallest eddy size on the Kolmogorov scale (Middleman, 1974). Although these effects on the droplet diameter have been formulated empirically (Paul et al., 2004), the relationships are complicated and have not been generalized. Therefore, the effects of the physical properties of solutions are represented as a proportional constant $F$. From the slope of the data shown in Fig. 10, the values of $F$ for solutions C-1 and C-2 were determined to be 3000 and 1700, respectively. Finally, with Eq. (6) and the determined values of $F$, the following equation of the orifice-shaped micromixer for producing the desired droplet size has been obtained:

$$
D_{32}=F \cdot \varepsilon_{\text {jet }}^{-0.4}=7.7 F\left(\zeta \frac{u_{0}{ }^{3}}{d}\right)^{-0.4}
$$


Finally, the optimization methodology of the orifice-shaped micromixer is summarized. First, geometry of an orifice-shaped mixer is chosen, and the pressure drop and the droplet diameter obtained for various flow rates using a raw material solution are measured. From the measurement, the value of $\zeta$ for the geometry is determined as shown in Fig. 8. The value of $\varepsilon_{\text {jet }}$ is then calculated using Eq. (6). Next, the proportional constant $F$ of the raw material solution is obtained from the plot of the droplet diameter against $\varepsilon_{\text {jet }}$ as shown in Fig. 10. From the above procedure, the relationship between $\varepsilon_{\text {jet }}$ and the droplet diameter (i.e., Eq. (7)) is established. This relationship gives the orifice hole size and the operating conditions for a given droplet diameter.

\subsection{Validation of effectiveness of using energy dissipation rate as optimization index}

In Section 3.2, through the consideration on mechanism of droplet formation, the energy dissipation rate was identified as the key index in the optimization of micromixers for the emulsification process, and the optimization methodology was established using this index. Then, in order to verify the effectiveness of this index in other geometries and sizes of micromixers, an instantaneous precipitation of fine particles by mixing a solution and a poor solvent was examined.

Fig. 11 shows the particle size distribution obtained by using the orifice-shaped 
micromixer as an instance of the result. The mean particle diameter was $600 \mathrm{~nm}$, and the size distribution was narrow. Because of the advantages of a low pressure drop and applicability to high-throughput operations, we conclude that the orifice-shaped micromixer is suitable for the production of fine particles with instantaneous nucleation as well as emulsification.

Fig. 12 shows the volume average particle diameter, $D_{43}=\Sigma D_{i}^{4} / \Sigma D_{i}^{3}\left(D_{i}\right.$ : diameter of droplet $i$ ), obtained by the micromixers as a function of the energy dissipation rate given by Eq. (2). The particle diameter was expressed as a function of the energy dissipation rate irrespective of the channel geometry and size. The results lead to the conclusion that the energy dissipation rate is applicable as an optimization index in the production of fine particles with rapid nucleation. In the range of $\varepsilon \geq 10^{6} \mathrm{~W} \cdot \mathrm{kg}^{-1}$, the mean particle size was almost fixed. This tendency implied that convection dominated the mixing process and the supplied fluids split into fluid segments within a considerably short period of time in this region. The size of the fluid segment converged into the size of a turbulent eddy, that is, Kolmogorov scale $\lambda$. At $\varepsilon=1 \times 10^{6} \mathrm{~W} \cdot \mathrm{kg}^{-1}$, the scale was estimated to be $\lambda=1.7 \mu \mathrm{m}$ from the kinematic viscosity $=2 \times 10^{-6} \mathrm{~m}^{2} \cdot \mathrm{s}^{-1}$. The diffusive mixing time $t_{\mathrm{d}}$ for diffusivity $D=10^{-9}$ $\mathrm{m}^{2} \cdot \mathrm{s}^{-1}$ was estimated to be $3 \mathrm{~ms}$ from the relation $t_{\mathrm{d}}=\lambda^{2} / D$. Therefore, when the time required for the formation of small fluid segments through convective mixing was considerably shorter than $3 \mathrm{~ms}$, a narrow particle size distribution could be obtained. Under the experimental conditions, the convective mixing time in the volume $V$ was approximately $1 \mathrm{~ms}$, and the 
convective mixing time, which is also the timescale of local nucleation, was sufficiently shorter than the time required for diffusive mixing. Summarizing above discussion, we concluded that the mixing proceeded under convection-controlled conditions in the experiments, and the particle formation process was as follows: convective mixing was completed within 1-2 ms; then, diffusive mixing took 2-3 ms to complete along with nucleation; finally, the nuclei grew uniformly after the mixing was completed. From the results discussed in this section, we conclude that the operation of rapid multiphase mixing is verified to be quantitatively optimized on the basis of the optimization index, i.e., the energy dissipation rate. 


\section{Conclusions}

For establishing an optimization methodology of the emulsification process in microchannels, the relationship between the channel geometry of the orifice-shaped micromixers and the size of the formed droplets has been investigated. We have devised indices to quantify the effects of the channel geometry on droplet formation, and to develop an optimization methodology. The results indicate that channel geometries for flow contraction and expansion dominate the emulsification performance and that the pressure drop can be correlated with the mean droplet diameter irrespective of the geometry.

Then, the mechanism of droplet formation has been discussed in order to establish the optimization methodology of orifice-shaped micromixer. This mechanism is as follows: the kinetic energy given to a fluid by contraction is dissipated within milliseconds by the formation of a micrometer-sized jet flow, which we named "micro-jet." On the basis of this mechanism, we have formulated the energy dissipation rate $\varepsilon_{\mathrm{jet}}$ and found that the mean droplet diameter is proportional to $\varepsilon_{\text {jet }}{ }^{-0.4}$ irrespective of the channel geometry of the mixer. Using this formulation, we integrated the geometric parameters of the orifice and the physical properties of the solutions into the expression of $\varepsilon_{\text {jet }}$ in order to establish an optimization methodology of droplet formation. This methodology provides channel sizes and operating conditions for obtaining droplets with a given diameter.

From the entire discussion presented in this paper, the rapid dissipation of kinetic 
energy within the timescale of milliseconds is the most important with respect to the droplet production in microchannels. In other words, a large rate of change in pressure is required in producing fine droplets. Although achieving such a rate is usually difficult in conventional macroscale processes, a simple geometry of sudden contraction and expansion with channel miniaturization enables us to achieve this requirement. The proposed orifice-shaped micromixer is a useful candidate to meet this demand in a single device under high-throughput and low-pressure-drop conditions. On the basis of the established optimization methodology, the application of micromixers is expected to be extended to the precise optimization of a fine-particle production process and the control of rapid reactions. 


\section{References}

Baldyga, J., Bourne, J.R., Zimmermann, B., 1994. Investigation of mixing in jet reactors using fast, competitive-consecutive reactions, Chemical Engineering Science 49, $1937-1946$.

Bayer, T., Heinichen, H., Natelberg, T., 2000. Emulsification of silicon oil in water-comparison between a micromixer and a conventional stirred tank, In: Proceedings of the 4th International Conference on Microreaction Technology, Atlanta, GA, pp. 167-173.

Benz, K., Jäckel, K.-P., Regenauer, K.-J., Schiewe, J., Drese, K., Ehrfeld, W., Hessel, V., Löwe, H., 2001. Utilization of micromixers for extraction processes, Chemical Engineering \& Technology 24, 11-17.

Charpentier, J.-C., 2005. Process intensification by miniaturization, Chemical Engineering \& Technology 28, 255-258.

Davies, J.T., 1985. Drop sizes of emulsions related to turbulent energy dissipation rates, Chemical Engineering Science 40, 839-842.

Falk, L., Commenge, J.-M., 2010. Performance comparison of micromixers, Chemical Engineering Science 65, 405-411.

Freitas, S., Walz, A., Merkle, H.P., Gander, B., 2003. Solvent extraction employing a static micromixer: A simple, robust and versatile technology for the microencapsulation of 
proteins, Journal of Microencapsulation 20, 67-85.

Guichardon, P., Falk, L., 2000. Characterisation of micromixing efficiency by the iodide-iodate reaction system. Part I: experimental procedure, Chemical Engineering Science 55, 4233-4243.

Guichardon, P., Falk, L., Villermaux, J., 2000. Characterisation of micromixing efficiency by the iodide-iodate reaction system. Part II: kinetic study, Chemical Engineering Science $55,4245-4253$.

Kawakatsu, T., Kikuchi, Y., Nakazima, M., 1997. Regular-sized cell creation in microchannel emulsification by visual microprocessing method, Journal of American Oil Chemists' Society $74,317-321$.

Kockmann, N., Kiefer, T., Engler, M., Woias, P., 2006. Convective mixing and chemical reactions in microchannels with high flow rates, Sensors and Actuators B 117, 495-508.

Löb, P., Pennemann, H., Hessel, V., Men, Y., 2006. Impact of fluid path geometry and operating parameters on 1/1-dispersion in interdigital micromixers, Chemical Engineering Science 61, 2959-2967.

Macloughlin, P.F., Malone, D.M., Murtagh, U.T., Kieran, P.M., 1998. The effects of turbulent jet flows on plant cell suspension cultures, Biotechnology and Bioengineering 58, 595-604.

Mae, K., Maki, T., Hasegawa, I., Eto, U., Mizutani Y., Honda, N., 2004. Development of a 
new micromixer based on split/recombination for mass production and its application to soap free emulsifier, Chemical Engineering Journal 101, 31-38.

Maruyama, T., Uchida, J., Ohkawa, T., Futami, T., Katayama, K., Nishizawa, K., Sotowa, K., Kubota, F., Kamiya, N., Goto, M., 2003. Enzymatic degradation of p-chlorophenol in a two-phase flow microchannel system, Lab on a chip 3, 308-312.

Mastuyama, K., Mine, K., Kubo, H., Mae, K., 2008. Operation and optimization of microreactors using convective mixing for fabrication of organic microparticles, In: Proceedings of the International Symposium on Micro Chemical Process and Synthesis, Kyoto, pp. 166-167.

Middleman, S., 1974. Drop size distributions produced by turbulent pipe flow of immiscible fluids through a static mixer, Industrial \& Engineering Chemistry Process Optimization and Development 13, 78-83.

Nagasawa, H., Aoki, N., Mae, K., 2005. Optimization of a new micromixer for instant mixing based on the collision of micro segments, Chemical Engineering Technology 28, $324-330$.

Paul, E.L., Atiemo-Obeng, V.A., Kresta, S.M., 2004. Handbook of Industrial Mixing, John Wiley \& Sons, Hoboken, NJ.

Rajaratnam, N., 1976. Turbulent Jets, Elsevier Scientific Publishing Co., Amsterdam.

Utada, A.S., Lorenceau, E., Link, D.R., Kaplan, P.D., Stone, H.A., Weitz, D.A., 2005. 
Monodisperse double emulsions generated from a microcapillary device, Science 308, $537-541$.

Zumaeta, N., Byrne, E.P., Fitzpatrick, J.J., 2008. Breakage of protein precipitates flowing through orifices, Chemical Engineering Research and Optimization 86, 107-117. 


\section{List of figure and table captions}

Fig. 1. Geometry and the geometric parameters of the orifice-shaped micromixer.

Fig. 2. Schematic representation of the experimental setup.

Fig. 3. The channel geometries of the micromixers: tube-shaped micromixer composed of coaxial double-pipes, tubes, and their fittings and bend-shaped micromixer composed of two inlet channels and an outlet channel crossing the inlet channels at those meeting point. For the outlet channels of the micromixers, each cross-sectional shape is a circle and each inner diameter is $d_{\text {out }}[\mathrm{mm}]$. The unit of the values given in this figure is millimeters.

Fig. 4. Comparison of the droplet size distributions among the orifice-shaped micromixers listed in Table 1. (a) When solution C-2 was used and the pressure drop was 0.30-0.35 MPa. (b) When solution C-1 was used and the pressure drop was 0.48-0.55 MPa.

Fig. 5. Relationship between $D_{32}$ and $\Delta P$, obtained in the case of orifice-shaped micromixers No. 2-6 with various total flow rates and the two solutions.

Fig. 6. Schematic representation of jet flow. A jet injected from the orifice is subjected to a shear force from the surrounding fluid, resulting in the generation of a local convective eddy inside of the jet. The jet is divided into the following two regions: the near-field and the developed-field. A potential core in which the flow is not subjected to shear exists inside of the near-field.

Fig. 7. Definition of the volume of space in which shear is applied, $V_{\text {jet }}\left[\mathrm{m}^{3}\right]$. 
Fig. 8. Determination of the values of $\zeta$ for orifice-shaped micromixers No. 2-6.

Fig. 9. Sauter diameter, $D_{32}$, as a function of the energy dissipation rate, $\varepsilon_{\text {jet }}$, defined by Eq. (6) in a double-logarithmic plot of the data from Fig. 5 when solution C-2 was used.

Fig. 10. Effect of the composition of solutions on the relationship between $D_{32}$ and $\varepsilon_{\text {jet }}$ when orifice-shaped micromixers No. 3-6 were used.

Fig.11. Particle size distribution of 1-octadecanol fine particles obtained by the precipitation method with orifice-shaped micromixer No. 2 and the total flow rate at $8.8 \mathrm{~L} \cdot \mathrm{h}^{-1}$.

Fig. 12. Volume average particle diameter of 1-octadecanol fine particles obtained by the micromixers as a function of the energy dissipation rate given by Eq. (2) in which each volume of focused space, $V$, was calculated using the channel diameter and the length given in the legend of this figure.

Table 1. Geometric parameters and operating conditions of orifice-shaped micromixers.

Table 2. Compositions of solutions. 


\section{Figures and tables:}

Fig. 1.

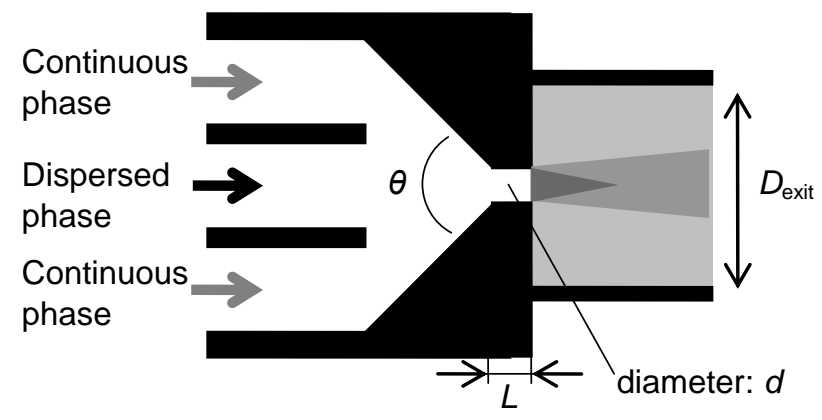


Fig. 2.

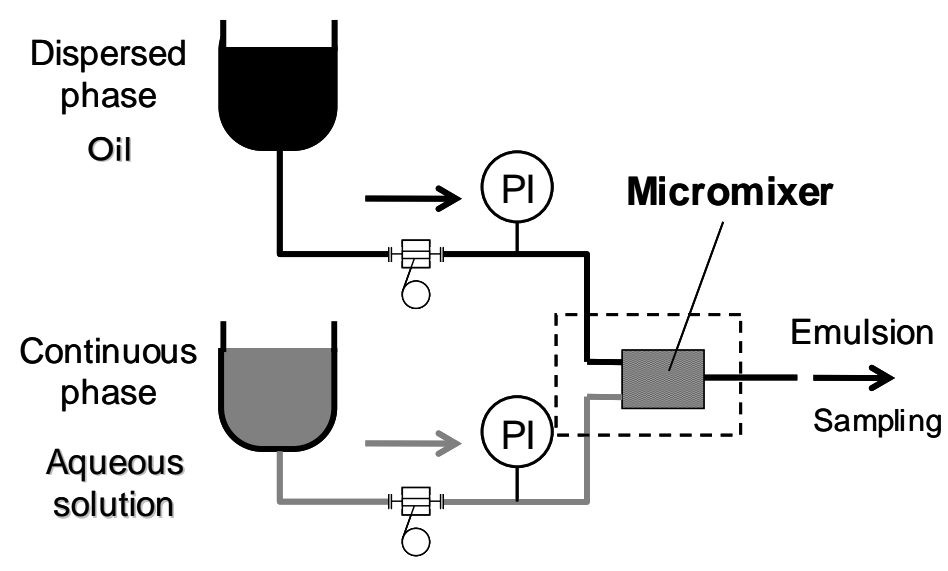


Fig. 3.

\section{Tube-shaped micromixer}

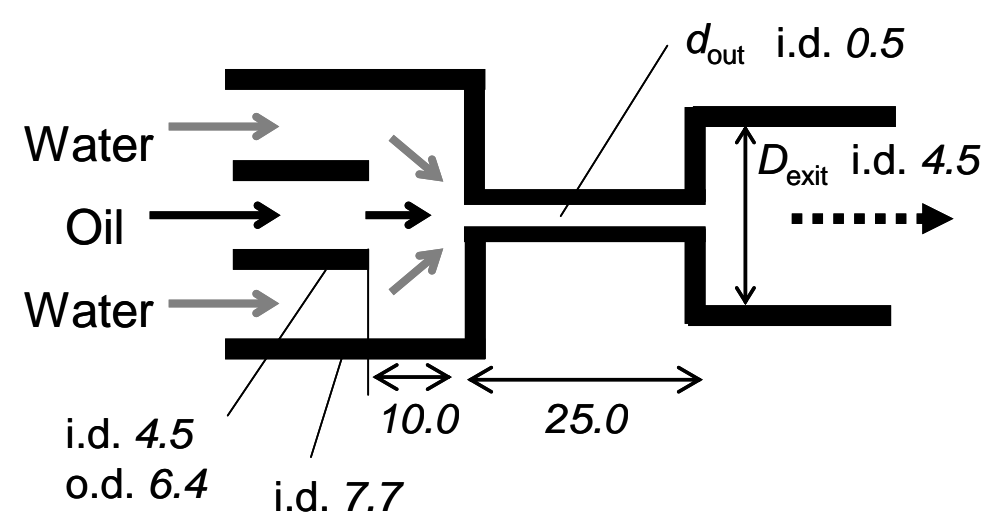

\section{Bend-shaped micromixer}

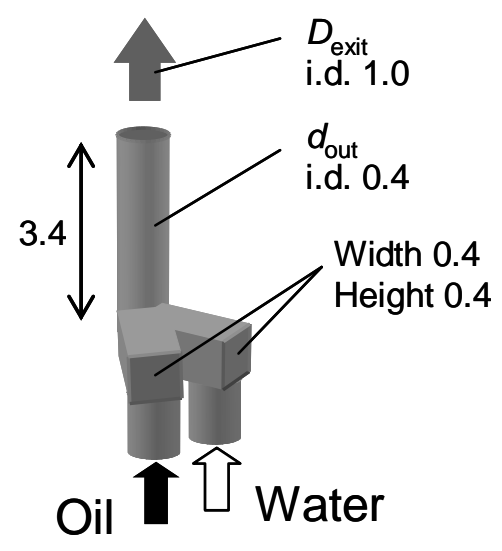


Fig. 4.

(a)

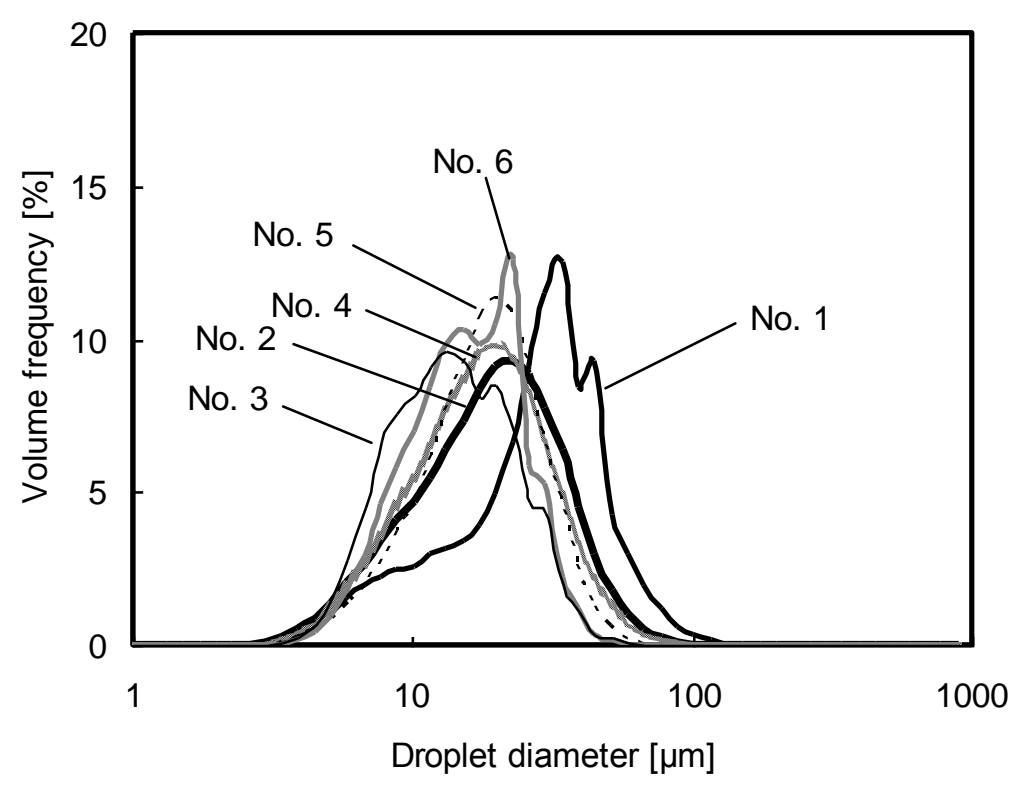

(b)

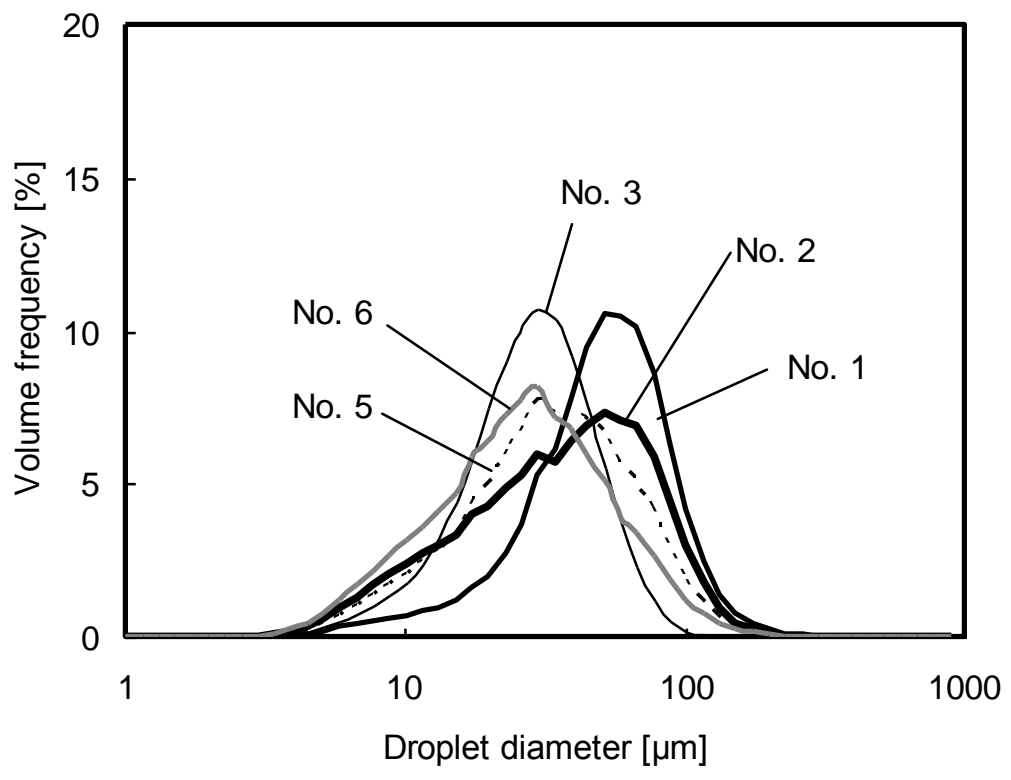


Fig. 5.

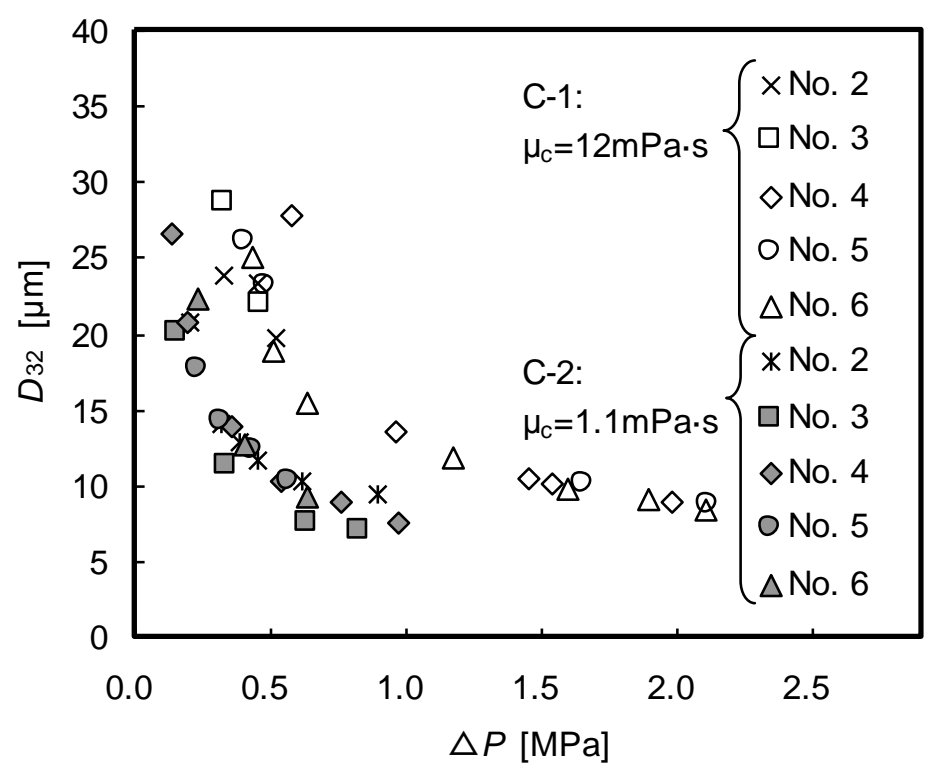


Fig. 6.

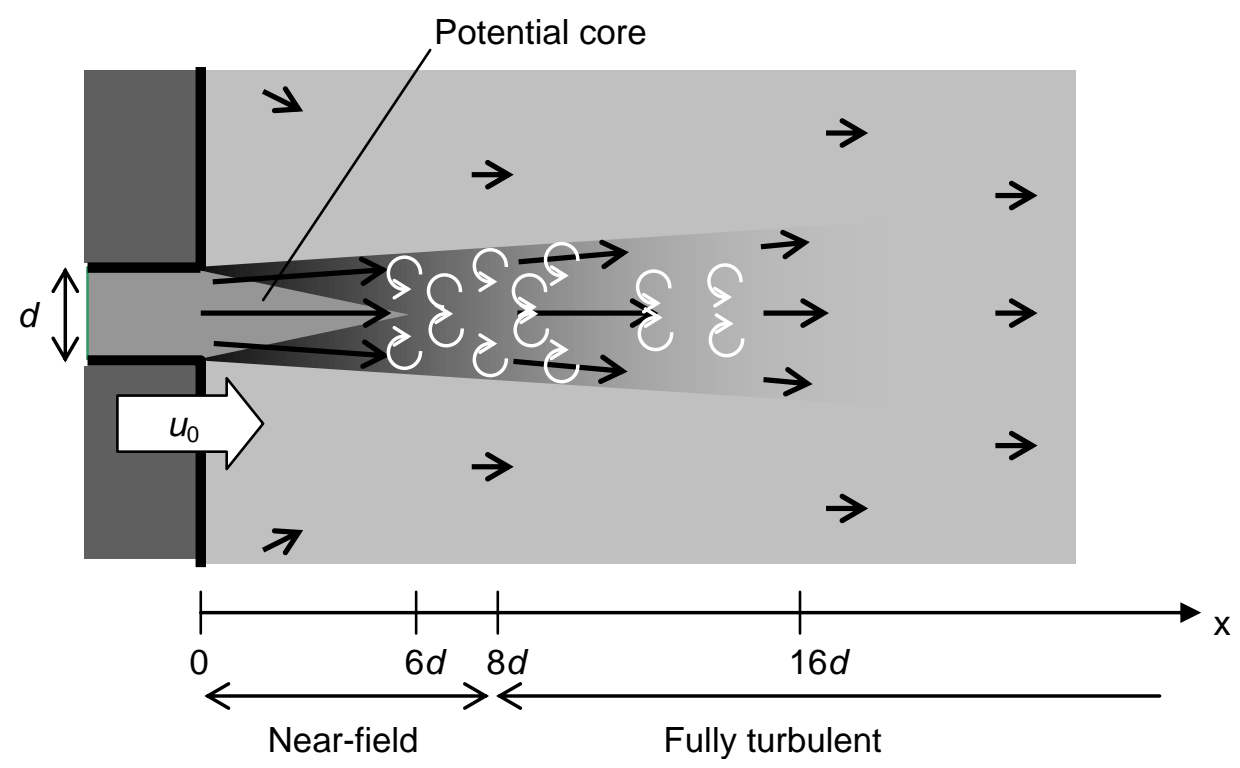


Fig. 7.

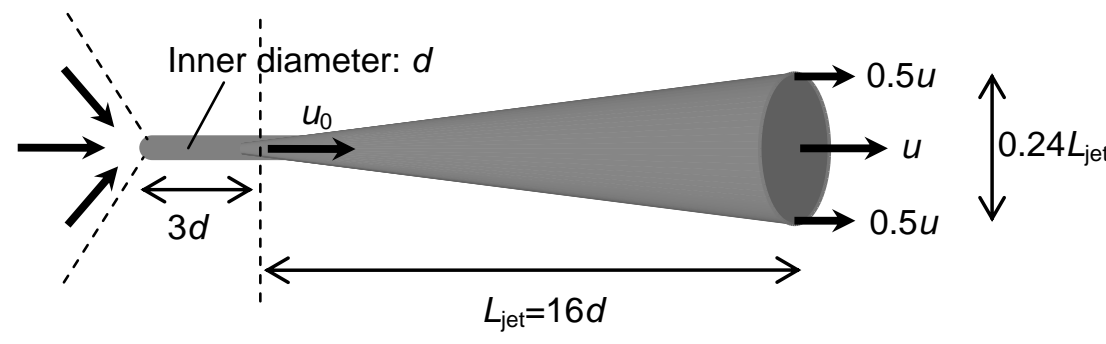

$$
\begin{aligned}
& \Rightarrow V_{\text {jet }}=81.6 d \cdot \pi d^{2} / 4 \\
& \Rightarrow \tau_{\text {jet }}=81.6 d / u_{0}
\end{aligned}
$$


Fig. 8.

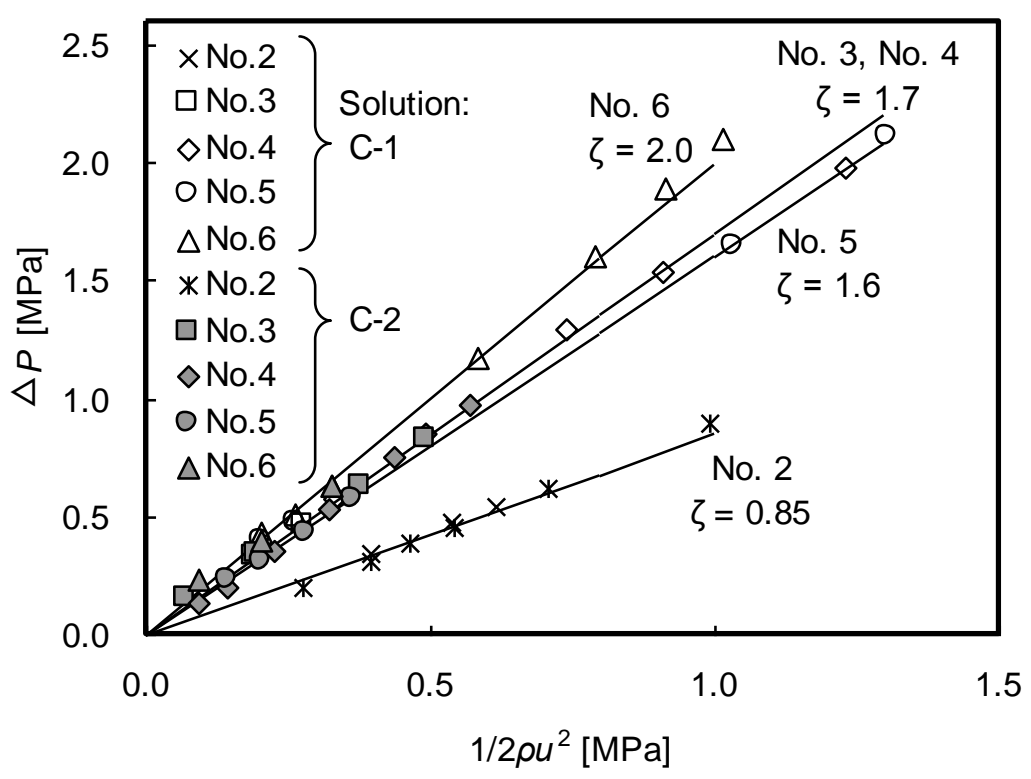


Fig. 9.

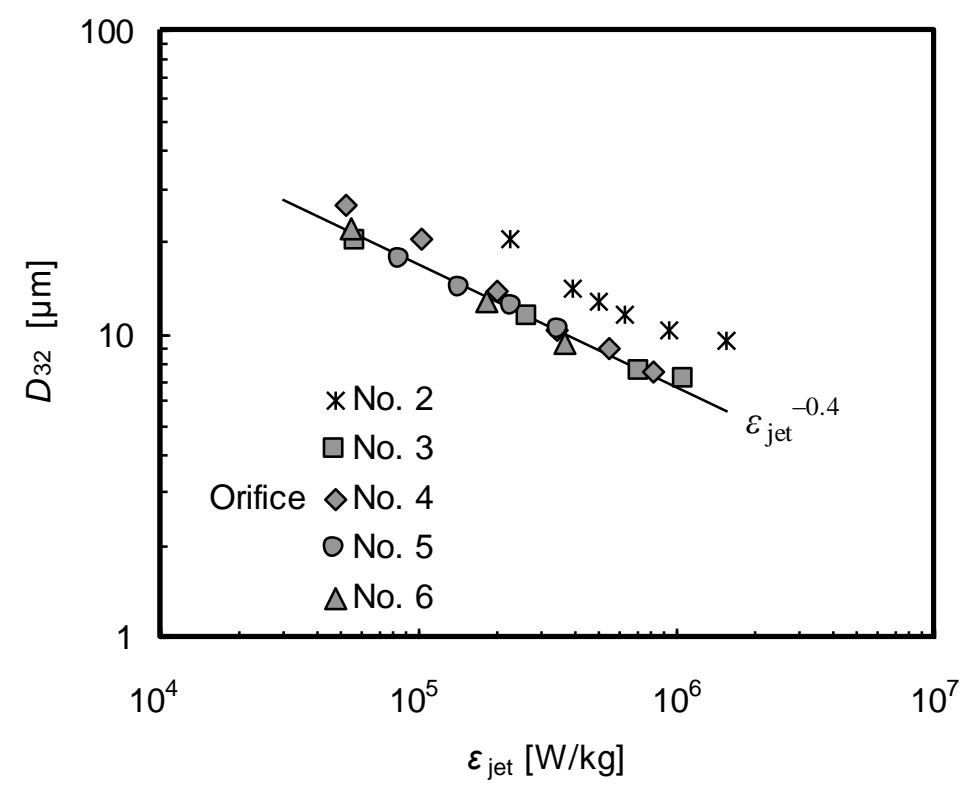


Fig. 10.

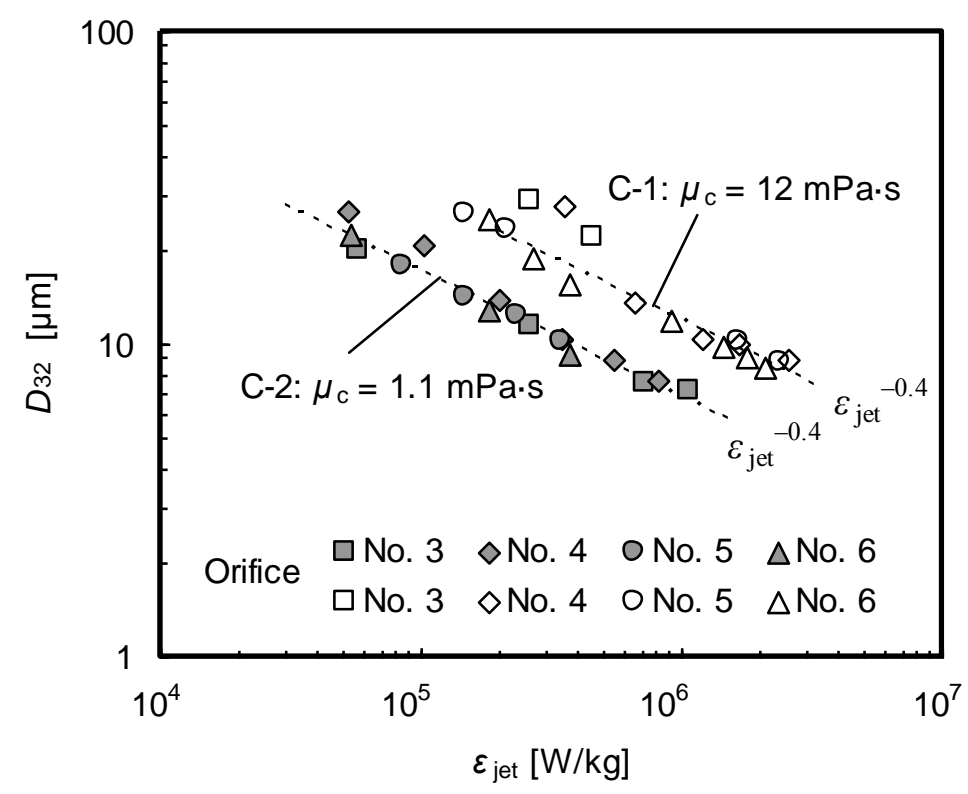


Fig. 11.

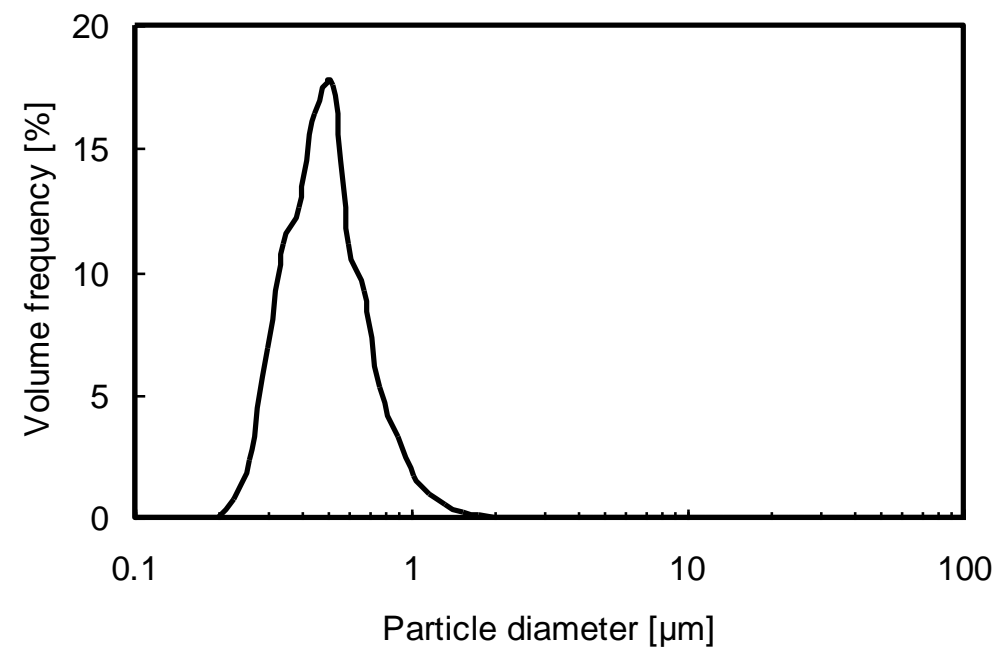


Fig. 12.

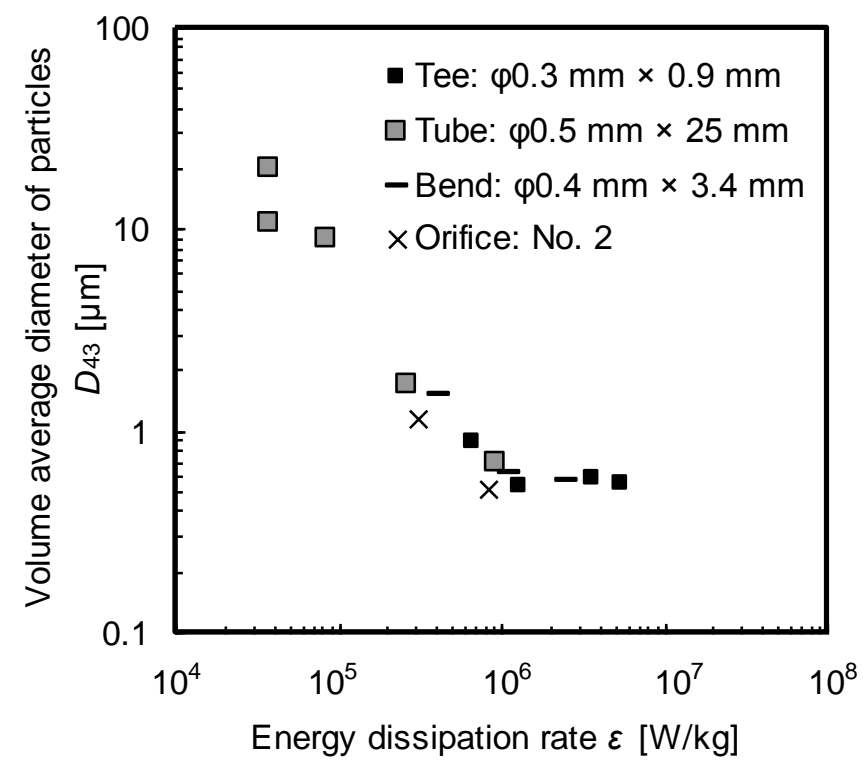


Table 1.

\begin{tabular}{|c|c|c|c|c|c|c|c|c|c|}
\hline No. & $\begin{array}{l}\text { Hole diameter } \\
\quad d[\mathrm{~mm}]\end{array}$ & $\begin{array}{l}\text { Hole length } \\
L[\mathrm{~mm}]\end{array}$ & $\begin{array}{c}\text { Angle } \\
\theta\end{array}$ & $\begin{array}{c}\text { Exit diameter } \\
D_{\text {exit }}[\mathrm{mm}]\end{array}$ & $\begin{array}{c}\text { Expansion ratio } \\
D_{\text {exit }} / d\end{array}$ & $\begin{array}{l}\text { Water phase } \\
\text { solution }\end{array}$ & $\begin{array}{l}\text { Volume flow } \\
\text { rate }[\mathrm{L} / \mathrm{h}]\end{array}$ & $\begin{array}{c}\text { Pressure drop } \\
\Delta P[\mathrm{MPa}]\end{array}$ & $\begin{array}{c}R e_{\text {hole }} \\
\text { (at hole) }\end{array}$ \\
\hline 1 & 0.3 & 0.9 & $180^{\circ}$ & 0.5 & 1.7 & $\begin{array}{l}\text { C-1 } \\
\text { C-2 }\end{array}$ & $\begin{array}{l}7.2-8.4 \\
7.2-9.6\end{array}$ & $\begin{array}{l}0.40-0.50 \\
0.32-0.63\end{array}$ & $\begin{array}{c}700-820 \\
7600-10000\end{array}$ \\
\hline 2 & 0.3 & 0.9 & $180^{\circ}$ & 1.1 & 3.7 & $\begin{array}{l}\mathrm{C}-1 \\
\mathrm{C}-2\end{array}$ & $\begin{array}{c}7.2-9.0 \\
6.0-11.4\end{array}$ & $\begin{array}{l}0.34-0.53 \\
0.20-0.90\end{array}$ & $\begin{array}{c}700-880 \\
6300-12000\end{array}$ \\
\hline 3 & 0.3 & 0.8 & $120^{\circ}$ & 7.0 & 23.3 & $\begin{array}{l}\mathrm{C}-1 \\
\mathrm{C}-2\end{array}$ & $\begin{array}{l}5.0-6.0 \\
3.0-8.0\end{array}$ & $\begin{array}{l}0.33-0.46 \\
0.15-0.83\end{array}$ & $\begin{array}{c}490-590 \\
3200-8500\end{array}$ \\
\hline 4 & 0.5 & 2.0 & $120^{\circ}$ & 18.0 & 36.0 & $\begin{array}{l}\text { C-1 } \\
\text { C-2 }\end{array}$ & $\begin{array}{l}18-35 \\
9.6-24\end{array}$ & $\begin{array}{l}0.58-1.98 \\
0.14-0.97\end{array}$ & $\begin{array}{c}1100-2100 \\
6100-15000\end{array}$ \\
\hline 5 & 0.56 & 1.7 & $60^{\circ}$ & 4.5 & 8.0 & $\begin{array}{l}\text { C-1 } \\
\text { C-2 }\end{array}$ & $\begin{array}{l}18-45 \\
15-24\end{array}$ & $\begin{array}{l}0.40-2.11 \\
0.23-0.57\end{array}$ & $\begin{array}{c}940-2400 \\
8500-14000\end{array}$ \\
\hline 6 & 0.56 & 1.7 & $120^{\circ}$ & 7.0 & 12.5 & $\begin{array}{l}\text { C-1 } \\
\text { C-2 }\end{array}$ & $\begin{array}{c}18-40 \\
12-22.8\end{array}$ & $\begin{array}{l}0.43-2.10 \\
0.23-0.63\end{array}$ & $\begin{array}{c}940-2100 \\
6800-13000\end{array}$ \\
\hline
\end{tabular}


Table 2.

\begin{tabular}{|c|c|c|c|}
\hline \multicolumn{2}{|c|}{ Compositon of solutions (wt $\%$ ) } & $\mathrm{C}-1$ & $\mathrm{C}-2$ \\
\hline \multirow{4}{*}{$\begin{array}{l}\text { Continuous } \\
\text { phase }\end{array}$} & Water & $69.3 \%$ & $79.6 \%$ \\
\hline & Polysaccharide & $0.11 \%$ & \\
\hline & Polyvinyl alcohol & $0.55 \%$ & $0.40 \%$ \\
\hline & Total & $70.0 \%$ & $80.0 \%$ \\
\hline $\begin{array}{l}\text { Dispersed } \\
\text { phase }\end{array}$ & Cyclic dimethyl silicone & $30.0 \%$ & $20.0 \%$ \\
\hline \multicolumn{2}{|c|}{ Physical properties of solutions (at $20^{\circ} \mathrm{C}$ ) } & $\mathrm{C}-1$ & $\mathrm{C}-2$ \\
\hline \multirow{2}{*}{$\begin{array}{c}\text { Continuous } \\
\text { phase }\end{array}$} & Viscocity $(\mathrm{mPa} \cdot \mathrm{s})$ & 12 & 1.1 \\
\hline & Dencity $\left(\mathrm{kg} / \mathrm{m}^{3}\right)$ & 998 & 998 \\
\hline \multirow{2}{*}{$\begin{array}{l}\text { Dispersed } \\
\text { phase }\end{array}$} & Viscocity (mPa.s) & \multicolumn{2}{|c|}{3.8} \\
\hline & Dencity $\left(\mathrm{kg} / \mathrm{m}^{3}\right)$ & \multicolumn{2}{|c|}{956} \\
\hline
\end{tabular}

